\title{
Acceso abierto a bases de datos para la generación de evidencias sobre recursos humanos en salud en el Perú
}

\section{Open access databases for generating evidence on human resources for health in Peru}

Correspondencia Alvaro Taype-Rondan alvaro.taype.r@upch.pe

Recibido: 30/10/2016 Aprobado: 16/11/2016

Citar como: Taype-Rondan A, Alva Díaz C, Jumpa-Armas D. Acceso abierto a bases de datos para la generación de evidencias sobre recursos humanos en salud en el Perú. Acta Med Peru. 2016;33(4):342-3

\author{
Alvaro Taype-Rondan ${ }^{1}$, Carlos Alva Díaz ${ }^{2,3,4}$, David Jumpa-Armas ${ }^{4,5}$ \\ 1 Centro de Excelencia en Enfermedades Crónicas, Universidad Peruana Cayetano Heredia. Lima, Perú. \\ 2 Servicio de Neurología, Hospital Nacional Daniel Alcides Carrión. Callao, Perú. \\ 3 Facultad de Medicina, Universidad Nacional Mayor de San Marcos. Lima, Perú. \\ 4 Red de Eficacia Clínica y Sanitaria (REDECS). Lima, Perú. \\ 5 Foro de la Sociedad Civil en Salud, Foro Salud. Lima, Perú.
}

\section{Sr. Editor:}

Distintas instituciones públicas y privadas poseen bases de datos potencialmente útiles para realizar investigaciones y generar evidencias que puedan ser utilizadas en la toma de decisiones. En la actualidad, la comunidad científica incentiva que estas bases de datos sean de acceso libre a cualquier persona, sin ninguna restricción, y con la información necesaria para ser analizada ${ }^{[1]}$. Esta accesibilidad se exige, en especial, para aquellas bases elaboradas con financiamiento público como el caso del portal "data.gov" que contiene numerosas bases de datos recolectadas por el gobierno de los Estados Unidos.

Al garantizar el libre acceso a estas bases de datos, se logra que individuos e instituciones externas diseñen sus propios análisis, generen nuevas evidencias, y contribuyan así en la toma de decisiones. Además, permite rehacer los análisis realizados por otros autores, lo cual transparenta el quehacer científico y permite la crítica y mejora de los métodos utilizados.

En Perú, la investigación en recursos humanos en salud es importante para poder plantear soluciones a sus problemas y fue establecida como una de las prioridades de investigación a nivel nacional para el periodo 2010-2014. Sin embargo, el número de publicaciones en este tema para dicho periodo fue muy reducido ${ }^{[2]}$.

Parte de esta problemática sería debido al limitado acceso a bases de datos sobre recursos humanos que dificulta su potencial uso para generar evidencias ${ }^{[3]}$. Este hermetismo contradice la política nacional de modernización de la gestión pública que tiene como eje transversal el Gobierno Abierto, definido como aquel que se abre al escrutinio público, accesible a los ciudadanos que lo eligieron, capaz de responder a sus demandas, necesidades y rendir cuentas de sus acciones y resultados ${ }^{[4]}$.

En el Perú, existen bases de datos que contienen información sobre recursos humanos en salud, siendo las más resaltantes las que exponemos en la Tabla 1. Dos de ellas son de acceso libre: la Encuesta Nacional de Satisfacción de Usuarios de Salud (ENSUSALUD), disponible desde la página del Instituto Nacional de Estadística e Informática (http://iinei.inei.gob.pe/microdatos/); y el sistema de Información 
Tabla 1. Bases de datos de entidades peruanas que contienen información sobre recursos humanos en salud.

Base de datos

Institución

Población y datos de interés

Año de última actualización

Encuesta Nacional de Satisfacción de Usuarios en Salud - ENSUSALUD*

Registro Nacional del Personal de la Salud - INFORHUS*

Encuesta a Establecimiento de Salud con Funciones Obstétricas y Neonatales

Encuesta a profesionales de salud que realizan el Servicio Rural y Urbano Marginal de Salud (SERUMS)

Encuesta descriptiva: percepción sobre el proceso de formación de médicos residentes en el sistema nacional de residentado médico (SINAREME) del Perú
Encuesta dirigida a usuario interno y externo sobre la valoración de satisfacción de las Instituciones Prestadoras de Servicios de 2016 Salud.

Información sobre profesión, cargo, nivel remunerativo y otros de los recursos humanos en salud a nivel nacional, regional y local

Encuesta dirigida a personal de salud y

Ministerio de Salud del Perú cuenta, entre otros datos, con información sobre profesionales disponibles para realizar atenciones maternas y neonatales.

Ministerio de Salud

Encuesta virtual dirigida a los profesionales de del Perú la salud al terminar su SERUMS, que incluye datos sobre su experiencia y problemática

Desconocido

Consejo Nacional del Residentado Médico
Encuesta virtual aplicada a residentes peruanos, que evalúa la percepción sobre aspectos académicas y laborales.

* Bases de datos de acceso libre.

de Recursos Humanos (INFORHUS), disponible desde su página web (http://observatorio.inforhus.gob.pe/bdatos.html).

Este acceso ha permitido a investigadores externos analizar sus datos y publicar diversos artículos científicos.

Si bien la "Ley de transparencia y acceso a la información pública" especifica que "Toda persona tiene derecho a solicitar y recibir información de cualquier entidad de la Administración Pública", actualmente esto no contempla la solicitud de bases de datos, y por lo general se limita a la entrega de medidas de resumen (como promedios o frecuencias), que son poco útiles para realizar estudios analíticos.

En conclusión, la falta de acceso público a estas bases de datos (muchas de ellas creadas con presupuesto público) es una práctica incorrecta que dificulta la generación de evidencias para la toma de decisiones. Ante este panorama, las entidades que poseen estas bases de datos tienen la oportunidad de corregir este hermetismo y promover una cultura de transparencia, acorde a nuestra era.

\section{REFERENCIAS BIBLIOGRÁFICAS}

1. Organisation for Economic Co-operation and Development. OECD Principles and Guidelines for Access to Research Data from Public Funding [Internet]. Paris: OECD; 2007 [citado el 16 de agosto de 2016]. Disponible en: http://www.oecd.org/sti/sci-tech/38500813. pdf

2. Oficina General de Investigación y Transferencia Tecnológica, Instituto Nacional de Salud. Prioridades Nacionales de Investigación en Salud 2015 - 2021. Resumen Ejecutivo. Lima, Perú: Instituto Nacional de Salud; 2015.

3. Fiestas F, Catacora MM. Uso público de bases de datos existentes como medida para potenciar la producción científica en salud en el Perú. Rev Peru Med Exp Salud Publica. 2010;27(4):652-3.

4. Presidencia del Consejo de Ministros de Perú. Decreto Supremo 004-2013-PCM. Aprueba la Política Nacional de Modernización de la Gestión Pública [Internet]. Lima, Perú: PCM; 2013 [citado el 18 de septiembre de 2016]. Disponible en: http://sgp.pcm.gob. pe/wp-content/uploads/2015/06/DS-004-2013-PCM-Aprueba-laPNMGP.pdf 